\title{
Multiscale isogeometric topology optimization for lattice materials
}

\author{
Yingjun Wang ${ }^{\mathrm{a}, \mathrm{b}}$, Hang $\mathrm{Xu}^{\mathrm{b}}$, Damiano Pasini ${ }^{\mathrm{b}, *}$ \\ ${ }^{a}$ School of Mechanical and Automotive Engineering, South China University of Technology, Guangzhou, 510640, China \\ ${ }^{\mathrm{b}}$ Department of Mechanical Engineering, McGill University, Montreal, Quebec, H3A0C3, Canada
}

\begin{abstract}
This paper presents isogeometric topology optimization (ITO) for periodic lattice materials, where non-uniform rational B-spline (NURBS) basis functions of CAD models are directly used in the finite element analysis to improve computational accuracy and efficiency. Two TO schemes that use asymptotic homogenization (AH) for the calculation of the mechanical properties are proposed for lattice materials with uniform and graded relative density respectively. To accelerate ITO for graded lattice materials, the mechanical properties are expressed as a function of the relative density of the unit cell, a step that avoids their iterative calculations during ITO. Three benchmark examples are presented to validate the proposed scheme with results that show tangible advantages, such as reduced computational time and faster convergence, of ITO over conventional TO.
\end{abstract}

Keywords: Topology optimization; Lattice material; Isogeometric analysis; Asymptotic homogenization; Multiscale mechanics

\section{Introduction}

Lattices are periodic materials that can be designed to obtain properties that primarily depend on the geometry of their repeating unit. The mechanical performance of lattice materials can be tailored to go beyond those of conventional materials, with structural advantages that are of interest in a large palette of applications, from aerospace lightweight components [1], to energy absorbing bumpers for vehicles [2], thermal insulation for civil engineering applications [3], as well as biomedical implants [4], among others. Compared to foams, generally characterized by a stochastic arrangement of cells, lattices allow better control of the cell arrangement, as they are generated by tessellating one porous unit along periodic vectors [5].

The mechanical properties of a lattice are mainly governed by the topology of the unit cell, whose characteristic length should be at least one order of magnitude below that of the component, should the periodic structure be considered as behaving as a material. Several theoretical approaches using the notion of Representative Volume Element have been proposed in the literature to calculate the mechanical properties of a lattice [6-9]. These schemes generally assume the unit cell walls behave like beams, following either Euler-Bernoulli or Timoshenko theory, and determine the elastic constants of the cell

\footnotetext{
*Corresponding Author: Email: damiano.pasini@mcgill.ca Phone: 514-398-6295 Fax: 514-398-7365
} 
through the classical solution of deformation and equilibrium problems. These approaches provide accurate results for values of relative density below 0.3 , above which beam theory looses accuracy. Furthermore, these theoretical approaches present limitations for unit cell with complex topology. On the other hand, homogenization methods (HMs), especially asymptotic homogenization (AH), have been proved to be able to rigorously predict the mechanical behavior of periodic materials [10-13]. In general, AH assumes that any field quantity can be described as an asymptotic expansion, which - replaced in the governing equations of equilibrium - allows to evaluate the effective properties of the material [14]. Since $\mathrm{AH}$ has neither limitation on the unit cell topology nor on the range of relative density, it has been widely used to calculate the properties of heterogeneous periodic materials [15-17].

Gradient based schemes for topology optimization (TO), such as the solid isotropic material with penalization (SIMP) [18, 19], evolutionary methods for structural optimization (ESO) [20, 21], as well as more recent strategies, such as the level set [22, 23], have been extended to optimize the internal architecture of porous materials or composites [24-29]. TO contributions exist in the literatures that address the design of either the macroscale geometry or the unit cell of lattice materials. For example, Niu et al. [30] presented a two-scale optimization method to maximize the fundamental frequency of cellular materials, where the mechanical properties of the unit cell were obtained via HM and were used as homogeneous properties to design the macroscopic domain of the material. Coelho et al. [31] presented a hierarchical framework for concurrent material and TO of 3D cellular structures, where the optimization scheme comprises two main loops. The outer deals with the macroscale design of the material, whereas the inner one uses HM for the TO of the unit cell design Nakshatrala et al. [32] proposed another multiscale framework to couple macro and micro TOs for nonlinear structural problems. Here, the design domain was partitioned into subdomains where the microstructure is imposed to remain uniform so as to ensure ease in fabrication. Despite this advantage, the work of Nakshatrala et al poses computational challenges due to the large computational power required to solve the coupled problem; hence the use of parallel computing and computer cluster was suggested [31, 32]. More recently, Khanoki et al [33] proposed a multiscale and multiobjective optimization for orthopedic hip implants with cellular material. In this work, the topology of the unit cell was predefined with mechanical properties expressed through fitting functions dependent on the relative density and directly used in the optimization loop, with the advantage of improved computational efficiency.

In recent years, isogeometric analysis (IGA) [34, 35], where the basis functions of the geometric model are directly used for finite element computations, has much attracted the attention of researchers in a variety of domains, as an efficient alternative to other conventional methods [36-42]. The high accuracy and efficiency of IGA provide a number of advantages over the established finite element method (FEM). For example, whereas checkerboards appearing in FEA can be easily relieved with higher order elements $[43,44]$, their use often requires high computational power [45]. IGA, on the other hand, can solve checkboard problems with much lower computational cost. Works that use IGA in structural optimization 
to capitalize on the IGA capability exist in literature, the first being the isogeometric TO (ITO) proposed by Seo et al. [46]. Later, Hassani et al. [47] proposed an isogeometric approach to TO where the so called control-point based SIMP was introduced to ease the interpolation of physical field quantities through the use of Non-uniform rational B-spline (NURBS). Dedè et al. [48] presented IGA for TO with a phase field model in both 2D and 3D problems, and demonstrated that IGA was particularly suitable for phase field problems as it allowed to handle exact CAD geometry. Very recently, Wang and Benson [49] proposed an ITO coupled with the level set method; here the NURBS basis functions of the CAD models are directly used for the parametrization of the level set functions and for the evaluation of the objective function. So far, however, all the ITO works existing in literature examine solid materials with isotropic properties. To the best of our knowledge, no work has so far extended ITO to porous materials, such as periodic lattices, with anisotropic properties.

In this paper, we present a framework that uses a multiscale ITO to optimize the relative density of lattice materials. Two schemes are proposed, one for homogeneous lattices and the other for graded lattices. AH is used to ease the calculation of the mechanical properties of lattice cell topologies through the use of curve fittings functions that are relative density dependent, thereby greatly improving the computation cost. The organization of the rest of this paper is as follows. Section 2 briefly introduces $\mathrm{AH}$ and the planar unit cell topologies that are examined in this work. Section 3 describes the NURBS based IGA for plane elasticity problems, while the multiscale ITO for lattice materials is proposed in Section 4. Thereafter three benchmark problems are presented in Section 5 to demonstrate the efficiency and accuracy of the proposed ITO method. Conclusions with future directions of research follow in Section 6.

\section{Mechanical properties of lattice materials}

\subsection{Asymptotic homogenization method}

Homogenization schemes $[10,50]$ have been widely used in elasticity to calculate the effective properties of a lattice material. Starting from the unit cell of a lattice, we can write its effective stress tensor $\bar{\sigma}_{i j}$ as a function of the strain tensor $\bar{\varepsilon}_{k l}$ through the effective elastic tensor $E_{i j k l}^{H}$ as

$$
\bar{\sigma}_{i j}=E_{i j k l}^{H} \bar{\varepsilon}_{k l}
$$

where $E_{i j k l}^{H}$ can be obtained via HM as follows. 


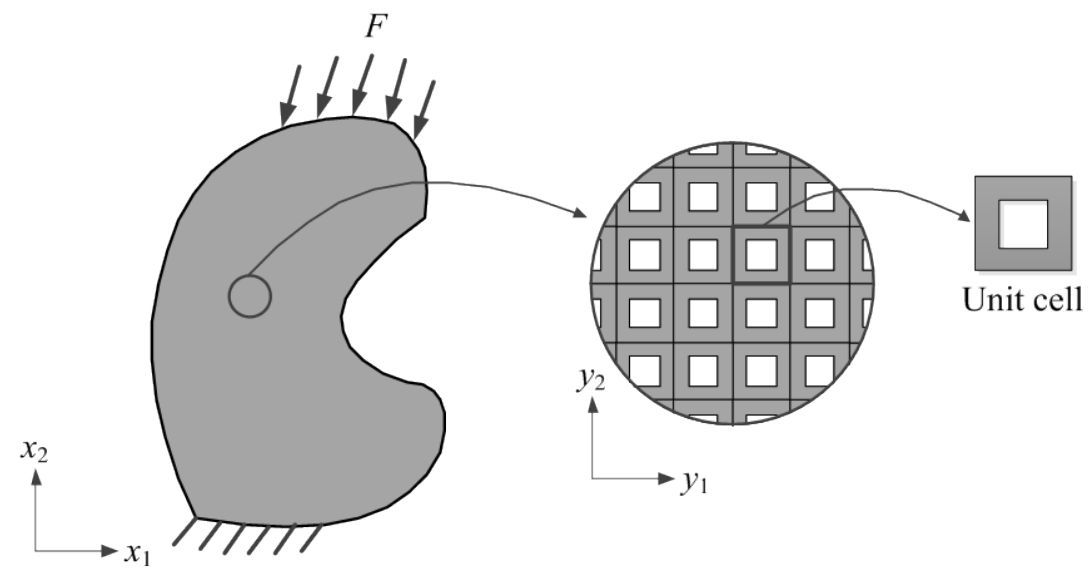

Figure 1. Illustration of a cellular domain consists of periodically patterned unit cells

Via asymptotic homogenization (AH) [14], we can express a physical field, e.g., the displacement field $u$, as an asymptotic expansion such that

$$
u^{\epsilon}(x)=u_{0}(x, y)+\epsilon u_{1}(x, y)+\epsilon^{2} u_{2}(x, y)+\cdots
$$

where $x$ and $y$ are the macroscopic and microscopic variables that are measured at the macroscale $(x)$ and at the microscale $(y)$ and $\epsilon=x / y$ is the aspect ratio of geometric features at the macro and micro scale. Generally $\epsilon$ is very small $(\epsilon \ll 1)$, and the functions $u_{0}(x, y), u_{1}(x, y) \ldots$ are $y$-periodic with respect to the local coordinate $y$.

If only the first order terms of the asymptotic expansion in Eq. (2) are considered, the effective stiffness tensor $E_{i j k l}^{H}$ can be calculated as

$$
E_{i j k l}^{H}=\frac{1}{|Y|} \int_{Y_{S}} E_{i j m n} M_{m n k l} d Y,
$$

where $|Y|$ is the volume of the entire unit cell with voids, $Y_{S}$ is the solid part of the cell, $E_{i j k l}$ is the local elasticity tensor which equals the elasticity tensor of the material in the solid domain of the cell and equals to zero for the void domain. $M_{i j k l}$ is the local structure tensor relating the macroscopic strain $\bar{\varepsilon}_{i j}$ to the microscopic strain $\varepsilon_{i j}$ as

$$
\varepsilon_{i j}=M_{i j k l} \bar{\varepsilon}_{i j}
$$

where $M_{i j k l}$ can be written as

$$
M_{i j k l}=\frac{1}{2}\left(\delta_{i k} \delta_{j l}+\delta_{i l} \delta_{j k}\right)-\varepsilon_{i j}^{* k l},
$$

where $\delta_{i j}$ is the Kronecker delta, and $\varepsilon_{i j}^{* k l}$ is the microstructural strain corresponding to the component $k l$ of the macroscopic strain tensor $\bar{\varepsilon}_{k l}$, and $\varepsilon_{i j}^{* k l}$ is obtained by solving a set of matrix equations; further details can be found in $[33,51]$. 


\subsection{Effective mechanical properties of four representative unit cells}

We select 4 planar topologies of the unit cell (see Figure 2) and use AH to calculate their mechanical properties as a function of the relative density. The results represent the homogenized properties of periodic lattices, each defined by a cell topology. In this study, each unit cell is assumed to have uniform strut thickness. The AH procedure is implemented in ANSYS (Canonsburg, Pennsylvania, U.S.A), where the $2 \mathrm{D}$ problem is built, meshed, and solved.

Since the planes of symmetry existing in a unit cell control the type of anisotropy of a lattice, we examine three types of symmetry that yield isotropic, orthogonal-isotropic, and orthotropic properties. Figure 2 shows them exemplified in four unit cells, each characterized by its own stiffness matrix and independent elastic terms: isotropic (hexagon), orthogonal-isotropic (square) and orthotropic (mixed triangular A and mixed triangular B). As can be seen, two, three and four are the independent constants required for the calculation of the terms of the stiffness matrix. The detailed expressions of these terms can be found in Eq. (16).

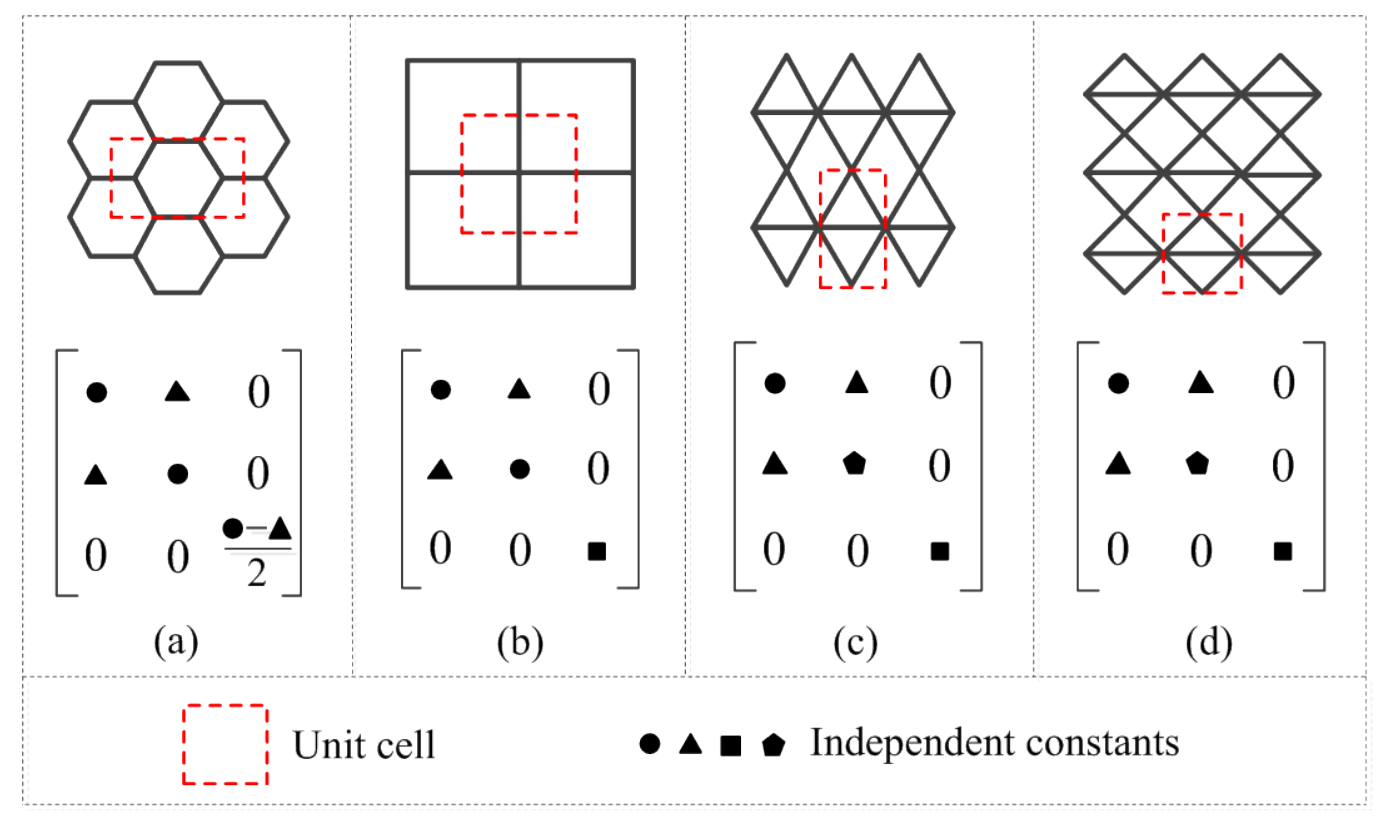

Figure 2. Unit cells and their schematic forms for the effective stiffness matrix: (a) hexagon, (b) square, (c) Mixed triangle A, and (d) Mixed triangle B.

Applying AH to the unit cells in Figure 2 with relative density from 0.1 to 1 allows to calculate their effective mechanical properties. Figure 3 shows the results, where $\bar{E}_{i i}, \bar{G}_{i j}$ and $\bar{v}_{i j}$ are the effective elastic modulus, shear modulus and Poisson's ratio of the lattice material, which are effective material properties obtained from the $\mathrm{AH}$, and $E_{S}$ and $v_{\mathrm{s}}$ are the elastic modulus and Poisson's ratio of the fully solid material that constructs the solid part of the unit cell. Figure 3 (a) and (b) show that the Young's moduli of hexagon and square lattices are equal in both the $x$ and $y$ directions; the difference is the shear modulus for the square lattice, which is an independent constant for orthogonal-isotropic materials. On the other hand, mixed triangle A and B are orthotropic, with Young's moduli different in both planar ( $x$-horizontal 
and $y$-vertical) directions. Comparing the effective mechanical properties of mixed triangle $\mathrm{A}$ and $\mathrm{B}$ in Figure 3 (c) and (d), we observe that $\bar{E}_{x}>\bar{E}_{y}$ for mixed triangle A (Mixed A for short) and $\bar{E}_{y}>\bar{E}_{x}$ for mixed triangle B (Mixed B), trends that confirm differences in mechanical properties between the unit cells examined in this work (Figure 2).

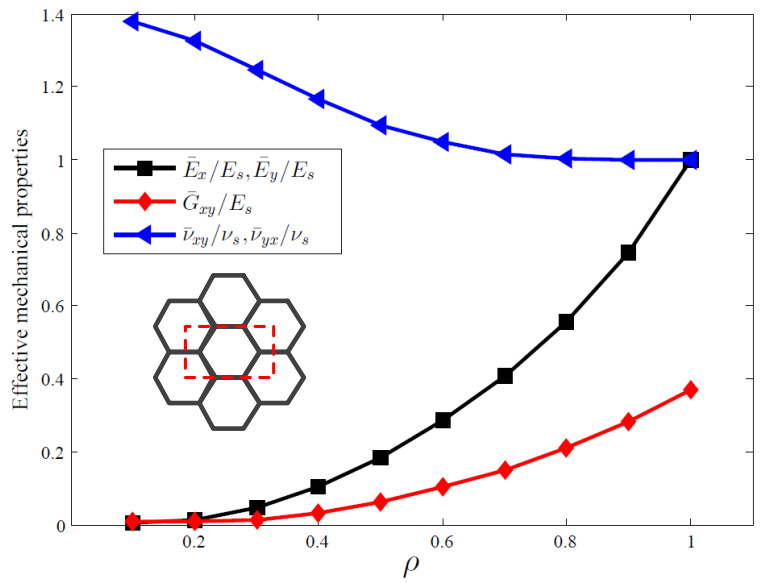

(a)

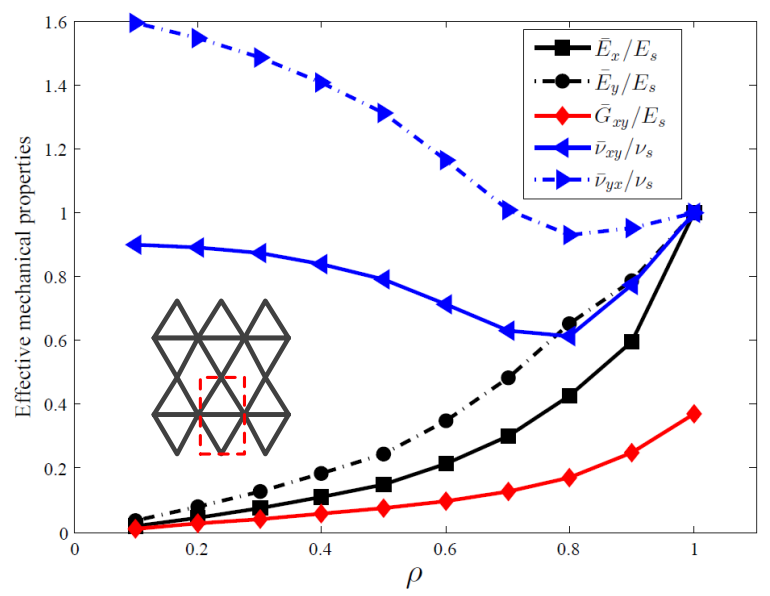

(c)

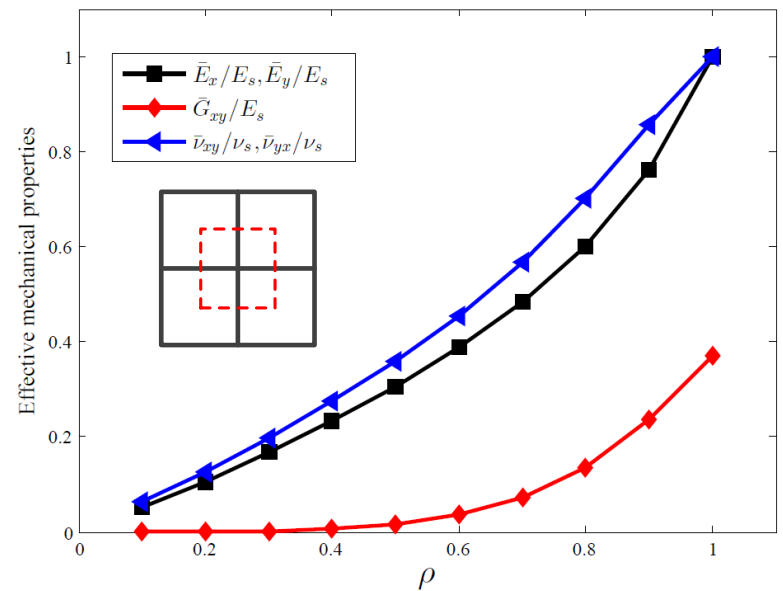

(b)

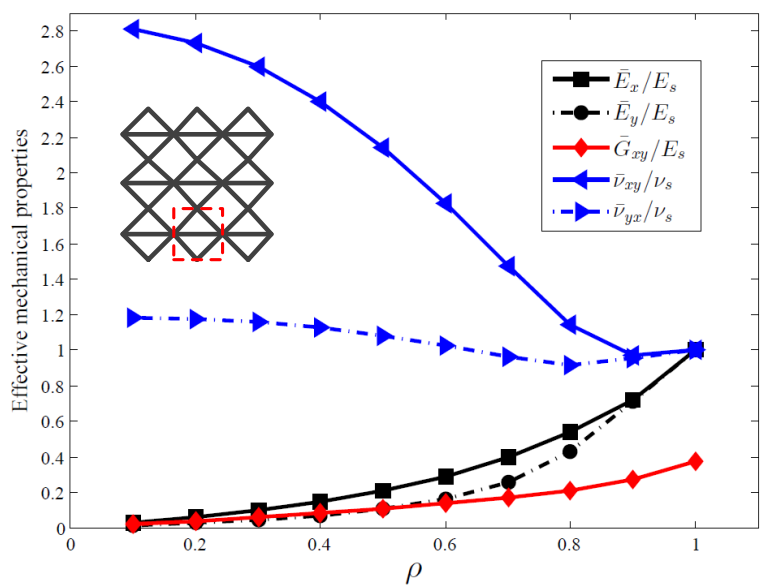

(d)

Figure 3. Effective mechanical properties as a function of relative density for (a) hexagon, (b) square, (c) mixed triangle $\mathrm{A}$, and (d) mixed triangle $\mathrm{B}$.

To obtain continuous relations from the discrete points describing the effective mechanical properties, we use the least squares for the relative density ranges of $\rho \leq 0.1$, and $0.1<\rho \leq 1$. Through these fitting functions, whose expressions are reported in the Appendix, the effective stiffness matrix corresponding to a given relative density can be directly calculated and promptly used in the optimization process. 


\section{Isogeometric analysis for planar elasticity problems of lattice materials}

\subsection{Summary of NURBS fundamentals}

Non-uniform rational B-splines (NURBS), constructed from B-splines, are commonly used in computer-aided design (CAD) and computer graphics $(\mathrm{CG})$ to generate and represent curves and surfaces [52]. A knot vector $\Xi=\left\{\xi_{1}, \xi_{2}, \ldots, \xi_{n+p+1}\right\}$, is a sequence of non-decreasing real numbers in the parametric space, where $n$ is the number of control points and $p$ is the order of the spline curve. The interval $\left[\xi_{1}, \xi_{n+p+1}\right]$ is called a patch and the knot interval $\left[\xi_{1}, \xi_{i+1}\right)$ is called a span.

Given a knot vector, the B-spline basis functions are recursively defined according to the Cox-de Boor formula [53]:

$$
\begin{gathered}
B_{i, 0}(\xi)=\left\{\begin{array}{l}
1, \text { if } \xi_{i} \leq \xi<\xi_{i+1}, \\
0, \text { otherwise }
\end{array}\right. \\
B_{i, p}(\xi)=\frac{\xi-\xi_{i}}{\xi_{i+p}-\xi_{i}} B_{i, p-1}(\xi)+\frac{\xi_{i+p+1}-\xi}{\xi_{i+p+1}-\xi_{i+1}} B_{i+1, p-1}(\xi), \quad(p>0) .
\end{gathered}
$$

By introducing a positive weight $w_{i}$ to B-spline basis functions, a NURBS basis function is defined as

$$
N_{i, p}(\xi)=\frac{B_{i, p}(\xi) w_{i}}{\sum_{j=1}^{n} B_{j, p}(\xi) w_{j}}
$$

Four important properties of NURBS basis functions are briefly listed here as: (1) Nonnegativity: $N_{i, p}(\xi) \geq 0$; (2) Partition of unity: $\sum_{i=1}^{n} N_{i, p}(\xi)=1$; (3) Local support: $N_{i, p}(\xi)=0$ for $\xi \notin\left[\xi_{i}, \xi_{i+p+1}\right)$; and (4) Differentiability: $N_{i, p}(\xi)$ is $p-k$ times differentiable where $k$ is the multiplicity of the knots.

According to the tensor product formulation, two-dimensional NURBS basis functions of order $p$ in $\xi$ direction and order $q$ in $\eta$ direction can be constructed as

$$
N_{i, p}^{j, q}(\xi, \eta)=N_{i, p}(\xi) N_{j, q}(\eta)
$$

and a NURBS surface is a bivariate piecewise rational function of the form

$$
S(\xi, \eta)=\sum_{i=1}^{n} \sum_{j=1}^{m} N_{i, p}^{j, q}(\xi, \eta) P_{i, j}
$$

where $P_{i, j}$ are the control points, and the patch for this surface is $\left[\xi_{1}, \xi_{n+p+1}\right] \times\left[\eta_{1}, \eta_{m+q+1}\right]$.

\subsection{Numerical implementation}

In contrast to conventional FEM, in isogeometric analysis (IGA) the numerical computations of a given physical field are calculated at the control points of the NURBS. Hence, a variable $x$ (e.g., coordinate, displacement, or force) whose parametric coordinate is $(\xi, \eta)$ can be evaluated from the control point values

$$
x(\xi, \eta)=\sum_{A} N_{A}(\xi, \eta) x_{A},
$$


where $N_{A}$ is the basis function of the $A$ th control point influencing the position of $(\xi, \eta)$, and $x_{A}$ is the corresponding value of the control point.

Figure 4 shows a comparison between NURBS elements and Lagrange elements, where the patch consists of 4 quadratic elements $(p=2)$ and the knot vectors in $\xi$ and $\eta$ both are $[0,0,0,0.5,1,1,1]$. Taking the left-bottom element as an example, we observe that the control points can be outside the NURBS element (Figure 4 (a)), whereas for a Lagrange element (Figure 4 (b)) all the nodes should fall within the element domain. Due to the support property of the NURBS basis functions $N_{i, p}(\xi) \neq 0$ for $\xi \in\left[\xi_{i}, \xi_{i+p+1}\right)$, the continuity, $C$, between the NURBS elements can reach $C^{p-1}$, while the continuity between the Lagrange elements is always $C^{0}$. As a result, the total number of control points in the NURBS mesh is much less than the number of nodes required in the Lagrange mesh (16 vs. 25 in this example), thereby demonstrating the need for fewer degrees of freedom (DOFs) in a NURBS mesh.

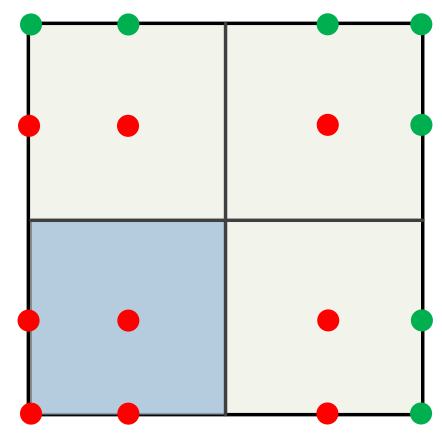

(a)

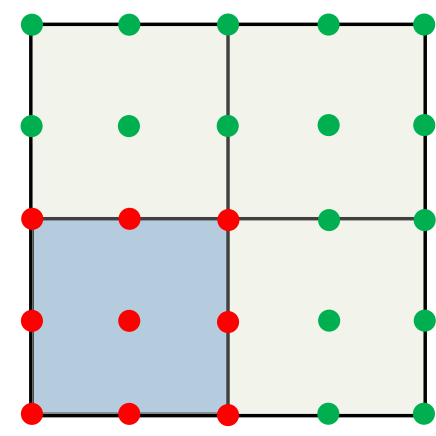

(b)

Figure 4. NURBS elements vs. Lagrange elements for a single-patch model: (a) NURBS elements and control points, and (b) Lagrange elements and nodes.

In IGA, the discrete equilibrium equation of a linear elasticity problem may be written in the form of the conventional FEM as [54]

$$
\boldsymbol{K u}=\boldsymbol{f},
$$

where $\boldsymbol{K}$ is the stiffness matrix, and $\boldsymbol{u}$ is the displacement vector and $\boldsymbol{f}$ is the external force vector associated with the control points. The stiffness matrix $\boldsymbol{K}$ is assembled by the element stiffness matrix $\boldsymbol{K}_{\boldsymbol{e}}$ which may be written as

$$
\boldsymbol{K}_{e}=\int_{\Omega_{e}} \boldsymbol{B}^{T} \boldsymbol{D} \boldsymbol{B} d \Omega=\int_{\widehat{\Omega}_{e}} \boldsymbol{B}^{T} \boldsymbol{D} \boldsymbol{B}\left|\boldsymbol{J}_{1}\right| d \hat{\Omega}=\int_{\bar{\Omega}_{e}} \boldsymbol{B}^{T} \boldsymbol{D} \boldsymbol{B}\left|\boldsymbol{J}_{1}\right|\left|\boldsymbol{J}_{2}\right| d \bar{\Omega},
$$

where $\boldsymbol{B}$ is the strain-displacement matrix and $\boldsymbol{D}$ is the material stiffness matrix, and $\Omega_{e}, \hat{\Omega}_{e}$ and $\bar{\Omega}_{e}$ are the physical, NURBS parametric and integration domains of the element, respectively. Jacobian $\boldsymbol{J}_{\mathbf{1}}$ and $\boldsymbol{J}_{2}$ indicate the transformation relationship that map integrals from the NURBS parametric space to the physical space, and from the integration parametric space to the NURBS parametric space.

In this study, we only consider 2D plane stress problems, for which the strain-displacement matrix $\boldsymbol{B}$ can be expressed as 


$$
\boldsymbol{B}=\left[\begin{array}{ccccc}
\frac{\partial N_{1}}{\partial x} & 0 & \cdots & \frac{\partial N_{n c}}{\partial x} & 0 \\
0 & \frac{\partial N_{1}}{\partial y} & \cdots & 0 & \frac{\partial N_{n c}}{\partial y} \\
\frac{\partial N_{1}}{\partial y} & \frac{\partial N_{1}}{\partial x} & \cdots & \frac{\partial N_{n c}}{\partial y} & \frac{\partial N_{n c}}{\partial x}
\end{array}\right]
$$

and

$$
\left[\begin{array}{ll}
\frac{\partial N_{i}}{\partial x} & \frac{\partial N_{i}}{\partial y}
\end{array}\right]=\left[\begin{array}{ll}
\frac{\partial N_{i}}{\partial \xi} & \frac{\partial N_{i}}{\partial \eta}
\end{array}\right] \boldsymbol{J}_{1}^{-1}
$$

where $N_{i}$ is the $i$ th basis function of the NURBS element and $n c$ is the number of control points per element, and the Jacobian $\boldsymbol{J}_{\mathbf{1}}$ is evaluated by

$$
\boldsymbol{J}_{\mathbf{1}}=\left[\begin{array}{ll}
\frac{\partial x}{\partial \xi} & \frac{\partial y}{\partial \xi} \\
\frac{\partial x}{\partial \eta} & \frac{\partial y}{\partial \eta}
\end{array}\right]
$$

The material stiffness matrix $\boldsymbol{D}$ of an orthogonal material may be written as

$$
\boldsymbol{D}=\left[\begin{array}{ccc}
\frac{E_{x}}{1-v_{x y} v_{y x}} & \frac{v_{y x} E_{x}}{1-v_{x y} v_{y x}} & 0 \\
\frac{v_{x y} E_{y}}{1-v_{x y} v_{y x}} & \frac{E_{y}}{1-v_{x y} v_{y x}} & 0 \\
0 & 0 & G_{x y}
\end{array}\right]=\left[\begin{array}{ccc}
D_{11} & D_{12} & 0 \\
D_{21} & D_{22} & 0 \\
0 & 0 & D_{33}
\end{array}\right],
$$

where $E_{i}$ is the Young's modulus along axis $i, G_{i j}$ is the shear modulus in the $j$ direction on the plane whose normal is in direction $i$, and $v_{i j}$ is the Possion's ratio that corresponds to a contraction in direction $j$ when an extension is applied along direction $i$. The above matrix $\boldsymbol{D}$ can be used for orthogonal-isotropic materials if $E_{x}=E_{y}$ and $v_{x y}=v_{y x}$, and for isotropic materials if $E_{x}=E_{y}, v_{x y}=v_{y x}$ and $G_{x y}=$ $E_{x} / 2\left(1+v_{x y}\right)$. It is worth noting that $D_{12}=D_{21}$.

The transformation from the Gauss quadrature domain to the NURBS parametric domain $\left[\xi_{i}, \xi_{i+1}\right] \times$ $\left[\eta_{j}, \eta_{j+1}\right]$ is linear as

$$
\left\{\begin{array}{c}
\xi=\frac{\xi_{i+1}-\xi_{i}}{2}(\bar{\xi}-1)+\xi_{i} \\
\eta=\frac{\eta_{j+1}-\eta_{j}}{2}(\bar{\eta}-1)+\eta_{j}
\end{array},\right.
$$

and therefore, the Jacobian $\boldsymbol{J}_{2}$ is defined as

$$
\boldsymbol{J}_{2}=\left[\begin{array}{ll}
\frac{\partial \xi}{\partial \bar{\xi}} & \frac{\partial \eta}{\partial \bar{\xi}} \\
\frac{\partial \xi}{\partial \bar{\eta}} & \frac{\partial \eta}{\partial \bar{\eta}}
\end{array}\right]=\left[\begin{array}{cc}
\frac{\xi_{i+1}-\xi_{i}}{2} & 0 \\
0 & \frac{\eta_{j+1}-\eta_{j}}{2}
\end{array}\right]
$$

For lattice materials, the effective mechanical properties, such as $E_{i}, G_{i j}$ and $v_{i j}$, are not only dependent on the constituent solid material, but also on the geometry of the unit cell, such as the cell topology and the strut thickness, which in turn controls the relative density. In this work, AH is used to establish a link between the properties at the microscale and those at the scale of the component. The 
relation between the two scales is used to first calculate the effective mechanical properties (see Section 2) and then to obtain the matrix $\boldsymbol{D}$. Since in the SIMP-based TO each unit cell corresponds to an element, the relative density of the unit cell directly correlates to the element relative density. Therefore, once the unit cell structure is selected and the mechanical properties are fitted as a function of the relative density, the multiscale TO can be implemented under the current TO scheme. More details on the process are provided in the following section.

\section{Multiscale isogeoemtric topology optimization of lattice materials}

\subsection{Sensitivity analysis}

\subsubsection{Basic statement of topology optimization}

The objective of a minimum compliance problem is to find the material density distribution that minimizes the deformation of a structure under prescribed support and loading conditions. The mathematical formulation of the optimization problem can be described as

$\min : c(\boldsymbol{\rho})=\boldsymbol{U}^{T} \boldsymbol{K} \boldsymbol{U}$

subject to: $\boldsymbol{K} \boldsymbol{U}=\boldsymbol{F}$

$$
\begin{aligned}
& V(\boldsymbol{\rho})=\boldsymbol{\rho}^{\boldsymbol{T}} \boldsymbol{v}=V_{m} \\
& \mathbf{0} \leq \boldsymbol{\rho} \leq \mathbf{1}
\end{aligned}
$$

where $c$ is the compliance, $\boldsymbol{K}$ is the global stiffness matrix, $\boldsymbol{U}$ and $\boldsymbol{F}$ are the global displacement and force vectors. $V(\boldsymbol{\rho})$ and $V_{m}$ are the material volume and volume constraint, and $\boldsymbol{\rho}$ and $\boldsymbol{v}$ are the element density and volume vectors, and the element densities that constitute $\boldsymbol{\rho}$ are the design variables.

The optimization problem can be solved by means of optimality criteria (OC). According to [55], a OC updating scheme can be formulated as

$$
\rho_{e}^{\text {new }}= \begin{cases}\max \left(0, \rho_{e}-m\right) & \text { if } \rho_{e} \beta_{e}^{\eta} \leq \max \left(0, \rho_{e}-m\right) \\ \min \left(1, \rho_{e}+m\right) & \text { if } \rho_{e} \beta_{e}^{\eta} \geq \min \left(0, \rho_{e}+m\right) \\ \rho_{e} \beta_{e}^{\eta} & \text { otherwise }\end{cases}
$$

where $m$ is a positive move limit, $\eta(=1 / 2)$ is a numerical damping coefficient, and $\beta_{e}$ is obtained from the optimality condition as

$$
\beta_{e}=\left(-\frac{\partial c}{\partial \rho_{e}}\right) /\left(\lambda \frac{\partial V}{\partial \rho_{e}}\right)
$$

where the Lagrange multiplier $\lambda$ is chosen with the bisection algorithm so that the volume constraint is satisfied.

\subsubsection{Sensitivity analysis via SIMP method}

For isotropic materials, the SIMP method can be used to define the effective Young's modulus of a mesh element [19] as 


$$
\bar{E}_{e}=\rho_{e}^{p} E_{0},
$$

where $E_{0}$ is the Young's modulus of the solid material, and $p$ is a penalization factor (typically $p=3$ ).

Based on Eq. (22), the sensitivity of the objective function $c$ with respect to the element density $\rho_{e}$ is

$$
\frac{\partial c}{\partial \rho_{e}}=-p \rho_{e}^{p-1} E_{0} \boldsymbol{u}_{e}^{T} \boldsymbol{k}_{\mathbf{0}} \boldsymbol{u}_{\boldsymbol{e}}
$$

where $\boldsymbol{u}_{\boldsymbol{e}}$ is the element displacement vector, and $\boldsymbol{k}_{\mathbf{0}}$ is the element stiffness matrix for an element with unit Young's modulus. When a uniform mesh is used and each element has unit volume, the sensitivity of the material volume $\partial V / \partial \rho_{e}$ equals to 1 .

However, Eqs. (22) and (23) are neither applied to orthotropic materials since the Young's modulus are different in $x$ and $y$ directions (see Eq. (16)), nor orthogonal-isotropic materials due to the independent shear modulus $G_{x y}$.

Assuming the effective element material stiffness matrix $\overline{\boldsymbol{D}}_{\boldsymbol{e}}$ is a function of the fully solid material stiffness matrix $\boldsymbol{D}$ and element density $\rho_{e}$ as [56]

$$
\overline{\boldsymbol{D}}_{\boldsymbol{e}}=\rho_{e}^{p} \boldsymbol{D}
$$

The SIMP can be extended to orthotropic materials when it is directly applied to the element stiffness matrix instead of the element Young's modulus in Eq. (22), as [29]

$$
\overline{\boldsymbol{k}}_{\boldsymbol{e}}=\rho_{e}^{p} \boldsymbol{k}_{\boldsymbol{e}}
$$

where $\overline{\boldsymbol{k}}_{\boldsymbol{e}}$ is the effective stiffness matrix of the element $e$ corresponding to the density $\rho_{e}$, and the $\boldsymbol{k}_{\boldsymbol{e}}$ is the stiffness matrix of the element $e$ with full solid material; the sensitivity of the objective function $c$ can be written as

$$
\frac{\partial c}{\partial \rho_{e}}=-\boldsymbol{u}_{\boldsymbol{e}}^{T} \frac{\partial \overline{\boldsymbol{k}}_{\boldsymbol{e}}}{\partial \rho_{e}} \boldsymbol{u}_{\boldsymbol{e}}=-p \rho_{e}^{p-1}\left(\boldsymbol{u}_{\boldsymbol{e}}^{\boldsymbol{T}} \boldsymbol{k}_{\boldsymbol{e}} \boldsymbol{u}_{\boldsymbol{e}}\right)=-\frac{p}{\rho}\left(\boldsymbol{u}_{\boldsymbol{e}}^{T} \overline{\boldsymbol{k}}_{\boldsymbol{e}} \boldsymbol{u}_{\boldsymbol{e}}\right)=-\frac{p}{\rho} c_{e}
$$

\subsubsection{Sensitivity analysis via fitting function}

SIMP works well for topology optimization problems with pure black-and-white solutions, i.e., the element densities are all 1 or 0 . Lattice materials, however, can be designed with continuous graded densities; their stiffness matrix is controlled by the effective material properties (obtained from the $\mathrm{AH}$ ), and thus it does not satisfy the relationship given in Eq. (24). Note that Eq. (26) may be still effective and used to update the new density in Eq. (20), but it is not the best as it gives only an approximate sensitivity of the objective function.

As described in Section 2.2, AH can be used to obtain the effective mechanical properties across a range of relative densities; the sensitivity of the objective function $c$ can be evaluated as

$$
\frac{\partial c}{\partial \rho_{e}}=-\boldsymbol{u}_{\boldsymbol{e}}^{\boldsymbol{T}} \frac{\partial \overline{\boldsymbol{k}}_{e}}{\partial \rho_{e}} \boldsymbol{u}_{\boldsymbol{e}}=-\boldsymbol{u}_{\boldsymbol{e}}^{\boldsymbol{T}} \frac{\partial \int_{\Omega_{e}} \boldsymbol{B}_{e}^{T} \overline{\boldsymbol{D}}_{\boldsymbol{e}} \boldsymbol{B}_{e} d \Omega}{\partial \rho_{e}} \boldsymbol{u}_{\boldsymbol{e}}
$$


From Eq. (16) and the fitting functions reported in the Appendix, we can find that each $\overline{\boldsymbol{D}}_{\boldsymbol{e}}$ component $D_{e_{-} i j}$ is a function of the element relative density $\rho_{e}$. Since $\boldsymbol{B}_{\boldsymbol{e}}$ is independent of $\rho_{e}$, Eq. (27) can be written as

$$
\frac{\partial c}{\partial \rho_{e}}=-\boldsymbol{u}_{e}^{T}\left(\int_{\Omega_{e}} \boldsymbol{B}_{e}^{T} \frac{\partial \overline{\boldsymbol{D}}_{e}}{\partial \rho_{e}} \boldsymbol{B}_{e} d \Omega\right) \boldsymbol{u}_{e}
$$

where $\frac{\partial \overline{\boldsymbol{D}}_{e}}{\partial \rho_{e}}$ can be obtained from the relationship between the fitting function of the effective mechanical properties and the relative density.

\subsection{Optimization procedure}

An ITO platform using MATLAB (Natick, Massachusetts, U.S.A) is developed in this work to solve the minimum compliance problem for both solid material and lattice material. In this platform, the standard SIMP which is used for isotropic solid material, is extended to handle lattice materials with both uniform and graded relative density.

Figure 5 illustrates the flow-diagram for ITO of lattice materials. Since isogeometric FEA is used, the meshing algorithm for conventional FEA is replaced by patch refinement [57], which avoids discretization errors. AH is used to obtain the effective mechanical properties of the unit cell, which are used during optimization. The sensitivity filter in [55] is used to avoid the checkerboard pattern. The convergence criterion here used is that the relative difference of the objective value between two iterations is less than $10^{-5}$, which is more strict than the value $10^{-4}$ previously used in literature [58]. It is worth noting that the extended SIMP method for lattice materials with only one density can be used for orthotropic (even anisotropic) solid materials by removing the AH part. 


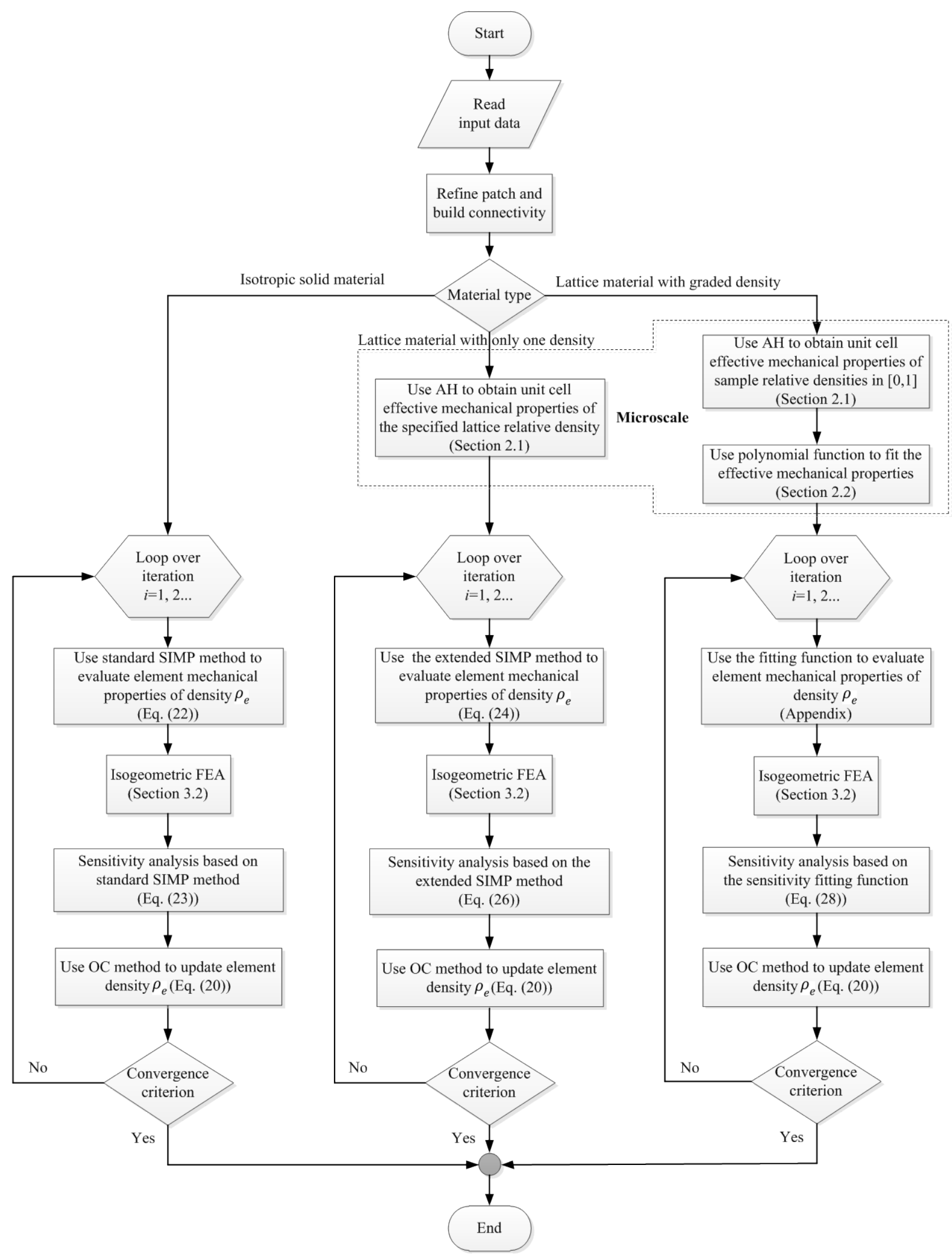

Figure 5. The flowchart of the multiscale ITO for isotropic solid material (left), uniform lattice (middle), and graded lattice (right). 


\section{Numerical examples}

Three benchmark examples for minimum compliance design of lattice materials are examined in this section with the goal of demonstrating the advantages of the ITO scheme presented in this work. All examples are run on a desktop computer with CPU Intel Xeon W3520 of $2.66 \mathrm{GHz}$, RAM of $16 \mathrm{~GB}$, and software environment MATLAB 2013a. The Young's modulus for the solid material is 1.0 and the Poisson's ratio is 0.35 . A Gauss quadrature rule of $3 \times 3$ is used for quadratic isogeometric elements.

In Section 5.1, we examine a classical Michell type structure to compare the computation efficiency of ITO versus the conventional FEM TO. In Section 5.2, we capture the role of cell topology in the optimal material distribution of a cantilever beam made of the lattices under investigation; finally Section 5.3 studies a half Messerschmidt-Bölkow-Blohm (MBB) beam to show the advantages of coupling ITO with AH for graded lattice materials.

\subsection{Michell type structure}

As a benchmark problem, we examine a simply supported beam under mid-span point load (Figure 6), commonly used to evaluate the merit of a given TO method [20, 59-61]. A fixed constraint is applied at the bottom-left corner and a roller constraint at the bottom-right corner.

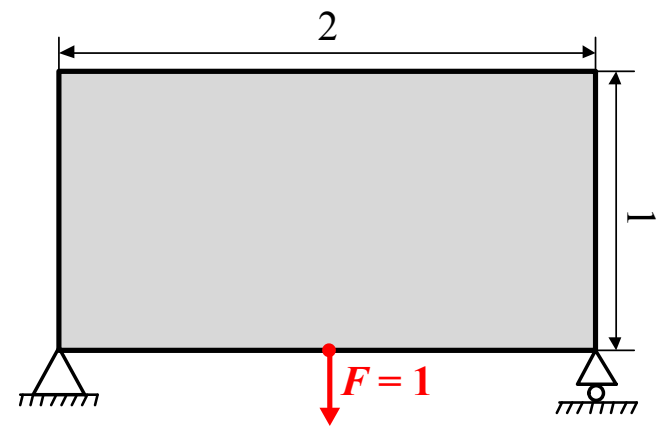

Figure 6. The design domain and boundary conditions of the classical Michell simply supported beam.

In this example, we aim to first compare the results obtained with ITO and conventional FEM TO. We use the optimization scheme for lattices with only one density (middle part of flow chart in Figure 5); the mechanical properties of the square unit cell (relative density $\rho_{c}$ is 0.8 ) are selected in the TO as those of the solid material (the elements whose element densities are 1). A mesh of $96 \times 48$ quadratic elements is used, and the volume ratio $\left(V R_{0}\right)$ is set to 0.5 , with $V R_{0}$ defined as

$$
V R_{0}=\frac{V_{m}}{V_{\text {total }}}
$$

where $V_{m}$ is the volume constraint in Eq. (19), and $V_{\text {total }}$ is the total volume of the design domain. For a lattice material, $V_{m}$ represents the domain that the lattice occupies, and the solid material volume corresponding to $V_{m}$ is 


$$
\bar{V}_{m}=\left(\boldsymbol{\rho}_{e} \circ \boldsymbol{\rho}_{c}\right)^{\boldsymbol{T}} \boldsymbol{v}
$$

where $\boldsymbol{\rho}_{\boldsymbol{e}}$ and $\boldsymbol{\rho}_{\boldsymbol{c}}$ are the element density and unit cell relative density, and $\circ$ is the element-wise product operation that multiplies vectors element by element. To describe the usage rate of the solid material, we define another volume ratio $(V R)$ as

$$
V R=\frac{\bar{V}_{m}}{V_{\text {total }}}
$$

with $V R=0.8 * V R_{0}=0.4$ for this example. This definition is also used in other examples hereinafter.

Figure 7 shows the results at selected optimization steps, with almost no difference between FEM TO and ITO. The number of iterations for the FEM and isogeometric TOs are 30 and 31, respectively. To compare the compliance in detail, we output the element density distribution of the IGA results, and use a conventional FEM to calculate the compliance. Although the difference is very small, the compliance of IGA results in each step is smaller than that of FEM results, which demonstrates that the high continuity of IGA can lead to higher accuracy. Recall that the convergence criterion (relative difference of the objective value between two iterations less than $10^{-5}$ ) results in a compliance of the IGA at step 31 , which is almost identical to that obtained at step 30 (i.e. 33.68). 


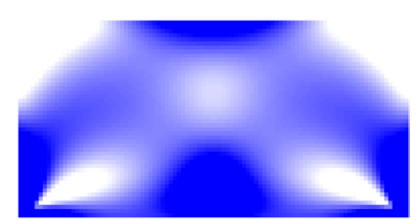

Step: 1 Compliance: 100.03

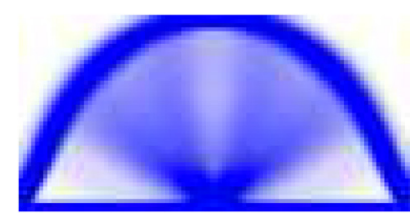

Step: 5 Compliance: 45.15

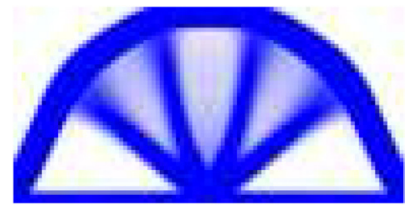

Step: 10 Compliance: 36.98

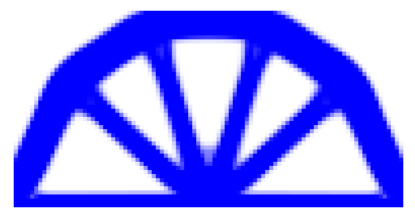

Step: 20 Compliance: 33.76

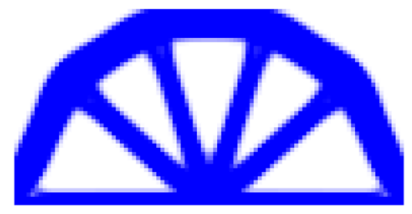

Step: 30 Compliance: 33.71

(a)

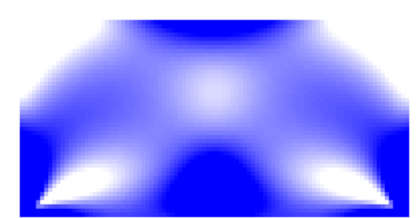

Step: 1 Compliance: 100.01

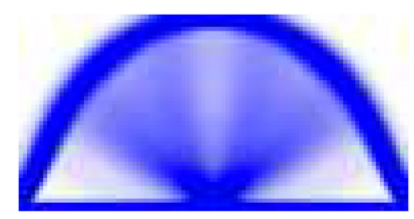

Step: 5 Compliance: 44.83

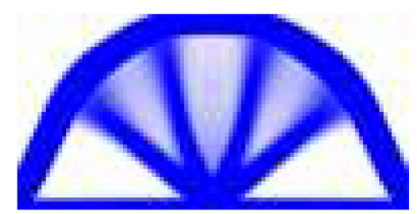

Step: 10 Compliance: 36.87

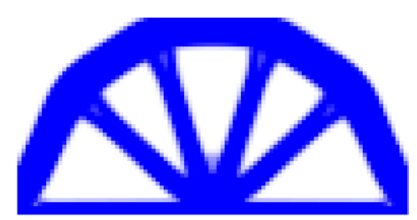

Step: 20 Compliance: 33.74

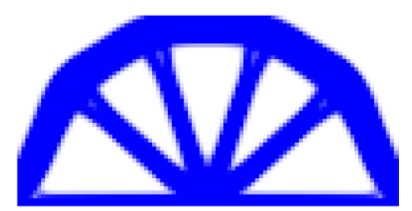

Step: 30 Compliance: 33.68

(b)

Figure 7. The optimization results: (a) FEM result and (b) IGA result. Average time at each step is 25.3s for FEM TO, and $8.01 \mathrm{~s}$ for ITO

One of the most important advantages of IGA is its high efficiency for high-order elements. According to [49], for a design domain with $e_{1} \times e_{2}$ elements, the DOFs of IGA and FEM using quadratic elements are

$$
N_{I G A}=2\left(e_{1}+2\right)\left(e_{2}+2\right) \text {, }
$$

and

$$
N_{F E M}=2\left(2 e_{1}+1\right)\left(2 e_{2}+1\right) .
$$

When $e_{1}$ and $e_{2}$ are large enough, $N_{F E M} / N_{I G A}$ is approximately 4. 
For the Michell type structure ( $96 \times 48$ quadratic elements), the DOFs of the FEM and IGA are 37442 and 9800 , respectively, and $N_{F E M} / N_{I G A}$ is 3.82 that approximates to 4 , which confirms the estimates from Eqs. (32) and (33). The average time at each step is $25.3 \mathrm{~s}$ for the FEM TO, and only $8.01 \mathrm{~s}$ for the ITO, and thereby the speedup is 3.16 .

To further discuss the computational efficiency of the method proposed in this work, we have modelled with quadratic elements selected meshes including $24 \times 12,48 \times 24,96 \times 48$ and $192 \times 96$, and compared the computational efficiency of TOs with IGA and FEM. In particular, the computational efficiency is here measured by the number of steps required to complete the whole computational procedure and the average time at each step, as shown in Table 1 . The results show that the ITO converges faster than the FEM TO, except for the $96 \times 48$ case where only one more step is needed with ITO. Furthermore, the speedup of IGA/FEM ranges from 2.29 to 3.30, values that prove the higher efficiency of the proposed ITO. We also emphasize that the computational cost for ITO and FEM TO are no longer linearly dependent on the DOFs, because some computations, such as element mechanical property evaluation, sensitivity analysis and design variable update, at each step are independent for the IGA or FEM.

Table 1

Comparison of computational efficiency of TOs, IGA and FEM.

\begin{tabular}{ccccccc}
\hline Cases & $N_{F E M} / N_{I G A}$ & $\begin{array}{c}\text { Number of } \\
\text { steps (FEM) }\end{array}$ & $\begin{array}{c}\text { Number of } \\
\text { steps (IGA) }\end{array}$ & $\begin{array}{c}\text { One step } \\
\text { time (FEM) }\end{array}$ & $\begin{array}{c}\text { One step } \\
\text { time (IGA) }\end{array}$ & $\begin{array}{c}\text { Speedups } \\
(\text { IGA/FEM) }\end{array}$ \\
\hline $24 \times 12$ & 3.37 & 33 & 32 & $0.48 \mathrm{~s}$ & $0.21 \mathrm{~s}$ & 2.29 \\
$48 \times 24$ & 3.66 & 31 & 26 & $3.14 \mathrm{~s}$ & $1.06 \mathrm{~s}$ & 2.96 \\
$96 \times 48$ & 3.82 & 30 & 31 & $25.3 \mathrm{~s}$ & $8.01 \mathrm{~s}$ & 3.16 \\
$192 \times 96$ & 3.91 & 77 & 67 & $402.6 \mathrm{~s}$ & $122.11 \mathrm{~s}$ & 3.30 \\
\hline
\end{tabular}

\subsection{Cantilever beam}

The second benchmark problem used to evaluate results of TOs is illustrated in Figure 8. [62-64]. The cantilever beam is fixed on the left-hand side and loaded with a vertical point force at the center of the right-hand side. A mesh of $96 \times 48$ quadratic elements is used for the computation. The volume ratio of optimization $\left(V R_{0}\right)$ is set to 0.5 for the lattice materials, and the relative density for the lattice materials $\left(\rho_{c}\right)$ is 0.6 , so as to obtain a volume ratio of solid material usage $(V R)$ of 0.3 . To compare the TO results for the lattice with those obtained with standard TO for an isotropic fully solid material, we separately examine two cases where the volume ratio of the solid material is 0.5 and 0.3 (with $V R_{0}=V R$ for the solid), 


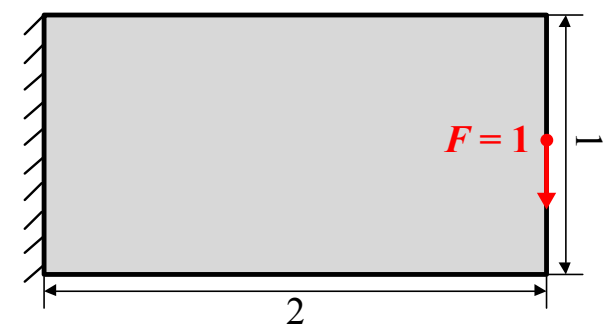

Figure 8 . The design domain and boundary conditions of the cantilever beam.

The main goal of this case study is to assess the role of cell topology in the TO results. We use for this reason a lattice material with uniform density. Figure 9 shows the TO results for the solid material and for the lattice materials examined in Section 2.2. From Figure 9 (c-f), we observe - as expected - that the planes of symmetries that each cell possesses control the material distribution with topological changes that differ between lattice cells. From Figure 9 (a-c), it can be found that the density distribution for the hexagon lattice almost replicates that obtained for a solid material with identical $V R_{0}$ but dissimilar $V R$, as both have isotropic properties; furthermore, the density distribution for the hexagon is different than that of a solid material for the identical $V R$ but different $V R_{0}$. These results infer that $V R_{0}$, as opposed to $V R$, is the parameter that control the element density distribution.

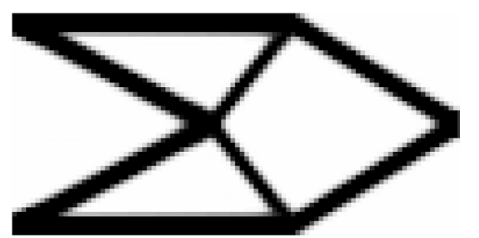

(a)

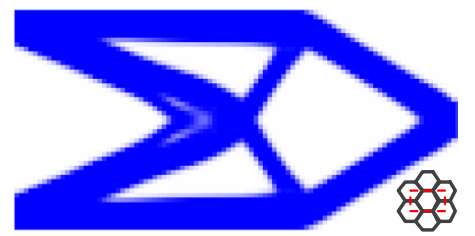

(c)

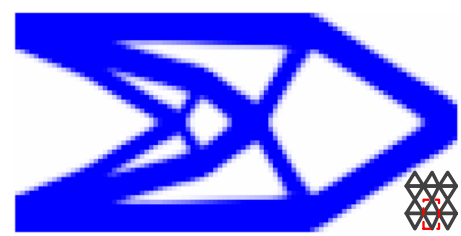

(e)

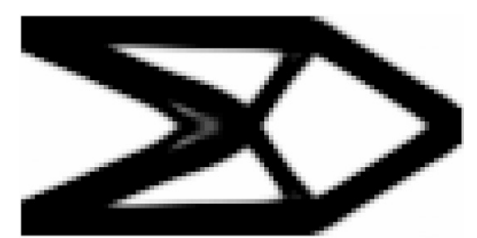

(b)

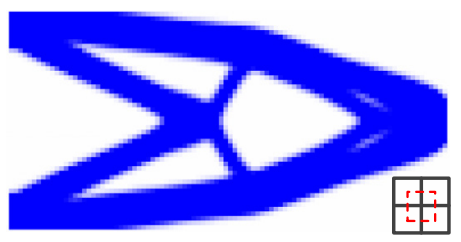

(d)

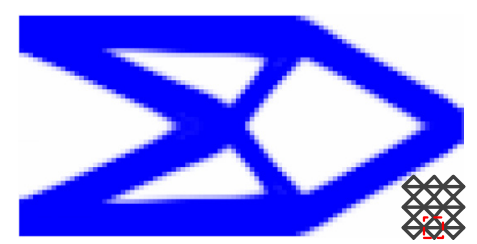

(f)

Figure 9. The optimization results of the cantilever beam: (a) solid with $\boldsymbol{V} \boldsymbol{R}=\mathbf{0 . 3}$, (b) solid with $\boldsymbol{V} \boldsymbol{R}=\mathbf{0 . 5}$, (c) hexagon lattice, (d) square lattice, (e) Mixed A lattice and (f) Mixed B lattice. Indicate here also what is VR for all the lattices $(\mathrm{VR}=3)$. You can write this info also in the figure if needed; else leave it in the caption.

Table 2 reports the compliance values and the number of iterations for the TO results illustrated in Figure 9. For given volume fraction $(V R=0.3)$, differences in compliance emerge among the candidate 
materials. As expected, the lattices are much softer than the solid material due to their higher porosity. For a higher material usage, the compliance would reduce for all the candidates, as demonstrated by the results for the solid material with $V R=0.3$ and $V R=0.5$. Furthermore, the mechanical properties of the materials have an influence also on the convergence of the TO solution. For example, a better convergence was achieved for isotropic materials, i.e. solid material and hexagon lattice, compared to the orthogonal-isotropic material (square lattice) and the orthotropic material (Mixed A and Mixed B).

Table 2

Compliance and number of iterations for the TOs corresponding to Figure 9.

\begin{tabular}{ccccccc}
\hline & $\begin{array}{c}\text { Solid } \\
(V R=0.3)\end{array}$ & $\begin{array}{c}\text { Solid } \\
(V R=0.5)\end{array}$ & $\begin{array}{c}\text { Hexagon } \\
(V R=0.3)\end{array}$ & $\begin{array}{c}\text { Square } \\
(V R=0.3)\end{array}$ & $\begin{array}{c}\text { Mixed A } \\
(V R=0.3)\end{array}$ & $\begin{array}{c}\text { Mixed B } \\
(V R=0.3)\end{array}$ \\
\hline $\begin{array}{c}\text { Compliance } \\
\text { Number of } \\
\text { iterations }\end{array}$ & 107.75 & 64.80 & 226.96 & 283.77 & 286.14 & 207.55 \\
\hline
\end{tabular}

\subsection{MBB beam}

Figure 10 shows the half Messerschmidt-Bölkow-Blohm (MBB) beam, another benchmark problem $[18,55,65]$. Due to symmetry, only half MBB beam is modelled and symmetry boundary conditions are applied. The design domain is discretized with $96 \times 32$ quadratic NURBS elements, and the volume ratio of optimization $\left(V R_{0}\right)$ is set to 0.5 . In this example, the goal of this case study is to assess the performance of ITO for lattice materials with graded density, where AH fitting functions are used to obtain the mechanical properties of the lattices; thus the volume ratio of the solid material usage $(V R)$ equals to the volume ratio of the optimization, i.e. $V R=V R_{0}=0.5$. To ensure the material in the design domain is a lattice, we limit the element relative density to the range $[0.05,0.95]$ in the whole TO.

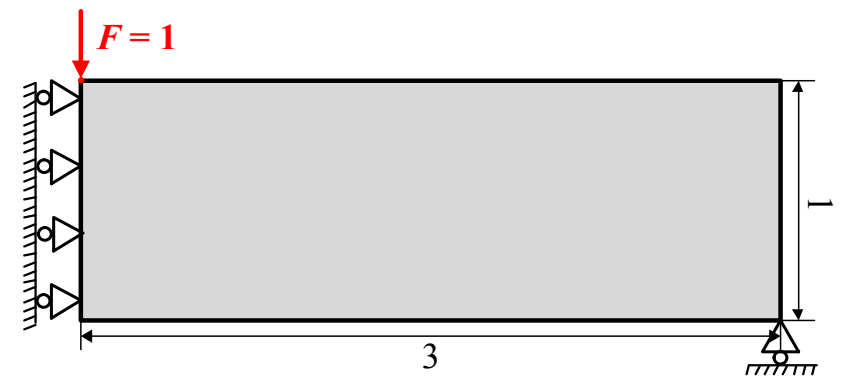

Figure 10. The design domain and boundary conditions of the half MBB beam.

To show the merit of the ITO scheme here proposed, we examine two lattice topologies, the hexagon (isotropic material) and the Mixed B (orthotropic material). Here, we use AH fitting functions to render the material property and Eq. (26) to calculate the sensitivity (call SenEq26 for short) with the goal of comparing the results with those obtained with Eq. (28) SenEq28. Figure 11 shows the optimization 
results of the half MBB beam; the topology of the hexagon lattice differs from that of the Mixed B lattice. The former reveals a black-and-white solution that is similar to that obtained with a conventional TO with a solid material, whereas the latter presents a grey domain that is generally avoided in a conventional TO with a solid material. The reason for this result is that the real relationship between lattice material properties and element relative density may be quite different from the hypothetical relationship used in the SIMP method (Eq. (22)), which may generate grey results. The material properties of the hexagon lattices differ from those of the Mixed B lattice (see Figure 3), a factor that explains the difference in the results shown in Figure $11(\mathrm{a}, \mathrm{b})$ and Figure $11(\mathrm{c}, \mathrm{d})$. This shows that the SIMP method is not suitable for the TO of graded lattices, $\mathrm{AH}$, on the other hand, can be used to calculate their mechanical properties and used in TO to obtain optimal density gradients in a prescribed domain, where each grey intensity describes a specific value of relative density for a given lattice.

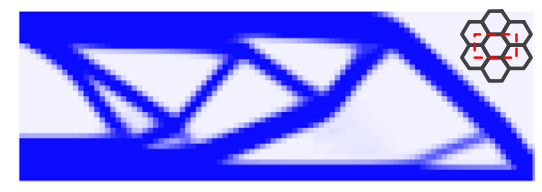

(a)

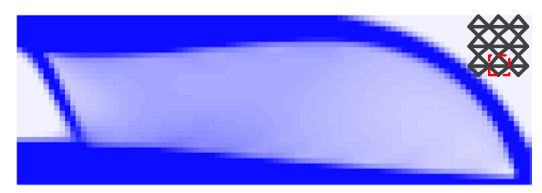

(c)

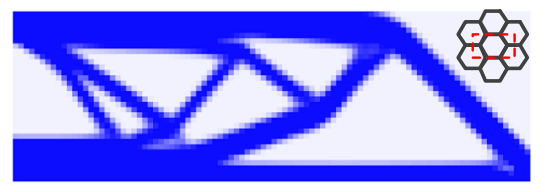

(b)

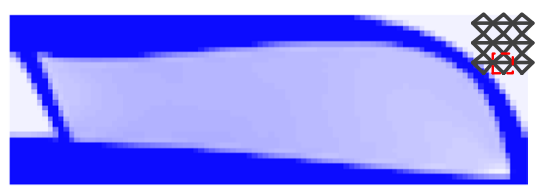

(d)

Figure 11. The optimization results of the half MBB beam: (a) hexagon lattice with SenEq26, (b) hexagon lattice with SenEq28, (c) Mixed B with SenEq26 and (d) Mixed B with SenEq28.

Table 3 shows the compliance values and the number of iterations for the TOs of the lattices shown in Figure 11. We can observe that the compliance from SenEq28 is slightly below that obtained with SenEq26, a result that is captured in Figure 11. This shows that the use of fitting functions to calculate the derivatives of the objective function leads to an accuracy higher than that obtained with the extended SIMP, thereby demonstrating the advantage of the proposed ITO scheme for the design of graded lattices.

Table 3

Compliance and number of iterations for the TOs (Figure 11).

\begin{tabular}{ccccc}
\hline & $\begin{array}{c}\text { Hexagon } \\
(\text { SenEq26) }\end{array}$ & $\begin{array}{c}\text { Hexagon } \\
(\text { SenEq28 })\end{array}$ & $\begin{array}{c}\text { Mixed B } \\
(\text { SenEq26) }\end{array}$ & $\begin{array}{c}\text { Mixed B } \\
(\text { SenEq28 })\end{array}$ \\
\hline Compliance & 223.64 & 221.84 & 221.46 & 219.68 \\
$\begin{array}{c}\text { Number of } \\
\text { iterations }\end{array}$ & 68 & 68 & 70 & 68 \\
\hline
\end{tabular}




\section{Conclusions}

This paper has presented an isogeometric TO (ITO) scheme for lattice materials with either isotropic, or orthogonal-isotropic, or orthotropic properties. For lattice materials with homogeneous density, we have extended the SIMP method to express the effective stiffness matrix of the element material as a function of the stiffness matrix of the solid material and the element density. For graded lattice materials, we have fitted the effective mechanical properties as a function of the unit cell relative density and directly use these functions in the TO iterations. The result shows improved computational efficiency. 3 case studies have been examined. The first has demonstrated a $1 / 3$ reduction of computational cost for ITO compared to FEM TO. The second has illustrated that the symmetry planes of each cell topology result in a specific relative density distribution. The third example for graded lattices has shown the merits (higher accuracy and better convergence) of coupling AH with ITO. Whereas the current work has focused on the density distribution only, further work is needed to guarantee unit cell connectivity. Concurrent optimization of unit cell topology and macroscale geometry, as well as the extension to 3D problems, are also part of future work.

\section{Appendix. Effective mechanical properties of unit cells in this study as a function of relative density}

Table 4 to Table 7 show the fitting functions used to represent the effective mechanical properties of the planar lattices examined in this work. Based on the least squares method, a linear interpolation is used for the relative density range of $[0,0.1]$ and a cubic polynomial function for relative density above 0.1 . Rsquared $\left(R^{2}\right)$ [67] are used to indicate how well data fit the function. An $R^{2}$ of 1 indicates that the function perfectly fits the data, whereas an $R^{2}$ of 0 indicates that the function does not fit the data at all.

\section{Table 4}

Effective mechanical properties of hexagon unit cell as a function of relative density

\begin{tabular}{cccc}
\hline & $\begin{array}{c}\text { Fitting function } \\
(\rho \leq 0.1)\end{array}$ & $\begin{array}{c}\text { Fitting function } \\
(0.1<\rho \leq 1)\end{array}$ & $\begin{array}{c}\text { R-squared } \\
\left(R^{2}\right)\end{array}$ \\
\hline$\frac{\bar{E}_{x}}{E_{s}}=\frac{\bar{E}_{y}}{E_{s}}$ & $0.066 \rho$ & $0.5824 \rho^{3}+0.3737 \rho^{2}+0.0432 \rho-0.0087$ & 0.9995 \\
$\frac{\bar{G}_{x y}}{E_{s}}$ & $0.06 \rho$ & $0.0845 \rho^{3}+0.389 \rho^{2}-0.1174 \rho+0.0137$ & 0.9998 \\
$\frac{\bar{v}_{x y}}{v_{s}}=\frac{\bar{v}_{y x}}{v_{s}}$ & $-0.5139 \rho+1.4287$ & $0.4921 \rho^{3}-0.2276 \rho^{2}-0.7177 \rho+1.4611$ & 0.9957 \\
\hline
\end{tabular}

\section{Table 5}

Effective mechanical properties of square unit cell as a function of relative density 


\begin{tabular}{|c|c|c|c|}
\hline 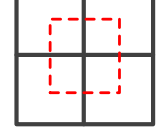 & $\begin{array}{l}\text { Fitting function } \\
\qquad(\rho \leq 0.1)\end{array}$ & $\begin{array}{l}\text { Fitting function } \\
(0.1<\rho \leq 1)\end{array}$ & $\begin{array}{c}\text { R-squared } \\
\left(R^{2}\right)\end{array}$ \\
\hline$\frac{\bar{E}_{x}}{E_{S}}=\frac{\bar{E}_{y}}{E_{S}}$ & $0.517 \rho$ & $1.2985 \rho^{3}-1.2525 \rho^{2}+0.986 \rho-0.0428$ & 0.9991 \\
\hline$\frac{\bar{G}_{x y}}{E_{s}}$ & $0.003 \rho$ & $1.0328 \rho^{3}-0.9154 \rho^{2}+0.2674 \rho-0.0209$ & 0.9996 \\
\hline$\frac{\bar{v}_{x y}}{v_{s}}=\frac{\bar{v}_{y x}}{v_{s}}$ & $1.9803 \rho+0.002$ & $0.2283 \rho^{3}+0.2524 \rho^{2}+0.5146 \rho+0.0115$ & 0.9997 \\
\hline
\end{tabular}

Table 6

Effective mechanical properties of Mixed A unit cell as a function of relative density

\begin{tabular}{cccc}
\hline & $\begin{array}{c}\text { Fitting function } \\
(\rho \leq 0.1)\end{array}$ & $\begin{array}{c}\text { Fitting function } \\
(0.1<\rho \leq 1)\end{array}$ & $\begin{array}{c}\text { R-squared } \\
\left(R^{2}\right)\end{array}$ \\
\hline$\frac{\bar{E}_{x}}{E_{s}}$ & $0.209 \rho$ & $2.7802 \rho^{3}-2.986 \rho^{2}+1.2678 \rho-0.0945$ & 0.9929 \\
$\frac{\bar{E}_{y}}{E_{s}}$ & $0.369 \rho$ & $0.348 \rho^{3}+0.4912 \rho^{2}+0.1368 \rho+0.0247$ & 0.9987 \\
$\frac{\bar{G}_{x y}}{E_{s}}$ & $0.129 \rho$ & $0.9649 \rho^{3}-1.063 \rho^{2}+0.4948 \rho-0.0314$ & 0.998 \\
$\frac{\bar{v}_{x y}}{v_{s}}$ & $-0.0979 \rho+0.9115$ & $4.3056 \rho^{3}-6.0366 \rho^{2}+1.9852 \rho+0.7328$ & 0.9624 \\
$\frac{\bar{v}_{y x}}{v_{s}}$ & $-0.4643 \rho+1.6402$ & $3.2887 \rho^{3}-5.0462 \rho^{2}+1.2585 \rho+1.4957$ & 0.9927 \\
\hline
\end{tabular}

Table 7

Effective mechanical properties of Mixed B unit cell as a function of relative density

\begin{tabular}{cccc}
\hline & $\begin{array}{c}\text { Fitting function } \\
(\rho \leq 0.1)\end{array}$ & $\begin{array}{c}\text { Fitting function } \\
(0.1<\rho \leq 1)\end{array}$ & $\begin{array}{c}\text { R-squared } \\
\left(R^{2}\right)\end{array}$ \\
\hline $\bar{E}_{x}$ & $0.277 \rho$ & $1.461 \rho^{3}-1.0976 \rho^{2}+0.6599 \rho-0.035$ & 0.9992
\end{tabular}




$$
\begin{array}{cccc}
\frac{\bar{E}_{y}}{E_{s}} & 0.117 \rho & 2.3064 \rho^{3}-1.9059 \rho^{2}+0.6493 \rho-0.0399 & 0.9987 \\
\frac{\bar{G}_{x y}}{E_{s}} & 0.19 \rho & 0.6203 \rho^{3}-0.6607 \rho^{2}+0.4267 \rho-0.0214 & 0.9984 \\
\frac{\bar{v}_{x y}}{v_{s}} & -0.8333 \rho+2.8961 & 6.2067 \rho^{3}-10.618 \rho^{2}+2.7732 \rho+2.5951 & 0.9965 \\
\frac{\bar{v}_{y x}}{v_{s}} & -0.082 \rho+1.1913 & 1.8941 \rho^{3}-2.9063 \rho^{2}+0.9008 \rho+1.1094 & 0.9825
\end{array}
$$

\section{References}

[1] J. Hohe, V. Hardenacke, V. Fascio, Y. Girard, J. Baumeister, K. Stöbener, J. Weise, D. Lehmhus, S. Pattofatto, H. Zeng, Numerical and experimental design of graded cellular sandwich cores for multifunctional aerospace applications, Materials \& Design, 39 (2012) 20-32.

[2] Q. Zhou, R.R. Mayer, Characterization of aluminum honeycomb material failure in large deformation compression, shear, and tearing, Journal of Engineering Materials and Technology, 124 (2002) 412-420.

[3] M.S. Al-Homoud, Performance characteristics and practical applications of common building thermal insulation materials, Building and environment, 40 (2005) 353-366.

[4] S.A. Khanoki, D. Pasini, Fatigue design of a mechanically biocompatible lattice for a proof-ofconcept femoral stem, journal of the mechanical behavior of biomedical materials, 22 (2013) 65-83.

[5] N. Fleck, V. Deshpande, M. Ashby, Micro-architectured materials: past, present and future, in: Proceedings of the Royal Society of London A: Mathematical, Physical and Engineering Sciences, The Royal Society, 2010, pp. 2495-2516.

[6] R.M. Christensen, Mechanics of cellular and other low-density materials, International Journal of Solids and Structures, 37 (2000) 93-104.

[7] A.-J. Wang, D. McDowell, In-plane stiffness and yield strength of periodic metal honeycombs, Journal of Engineering Materials and Technology, 126 (2004) 137-156.

[8] R.S. Kumar, D.L. McDowell, Generalized continuum modeling of 2-D periodic cellular solids, International Journal of Solids and Structures, 41 (2004) 7399-7422.

[9] A. Vigliotti, D. Pasini, Linear multiscale analysis and finite element validation of stretching and bending dominated lattice materials, Mechanics of Materials, 46 (2012) 57-68.

[10] J. Guedes, N. Kikuchi, Preprocessing and postprocessing for materials based on the homogenization method with adaptive finite element methods, Computer methods in applied mechanics and engineering, 83 (1990) 143-198.

[11] B. Hassani, E. Hinton, A review of homogenization and topology optimization I- homogenization theory for media with periodic structure, Computers \& Structures, 69 (1998) 707-717.

[12] A.L. Kalamkarov, I.V. Andrianov, V.V. Danishevsâ, Asymptotic homogenization of composite materials and structures, Applied Mechanics Reviews, 62 (2009) 030802.

[13] G.-D. Cheng, Y.-W. Cai, L. Xu, Novel implementation of homogenization method to predict effective properties of periodic materials, Acta Mechanica Sinica, 29 (2013) 550-556.

[14] S. Arabnejad, D. Pasini, Mechanical properties of lattice materials via asymptotic homogenization and comparison with alternative homogenization methods, International Journal of Mechanical Sciences, 77 (2013) 249-262.

[15] N. Takano, Y. Ohnishi, M. Zako, K. Nishiyabu, Microstructure-based deep-drawing simulation of knitted fabric reinforced thermoplastics by homogenization theory, International Journal of Solids and Structures, 38 (2001) 6333-6356.

[16] A. Visrolia, M. Meo, Multiscale damage modelling of 3D weave composite by asymptotic homogenisation, Composite Structures, 95 (2013) 105-113. 
[17] X. Peng, J. Cao, A dual homogenization and finite element approach for material characterization of textile composites, Composites Part B: Engineering, 33 (2002) 45-56.

[18] O. Sigmund, A 99 line topology optimization code written in Matlab, Structural and Multidisciplinary Optimization, 21 (2001) 120-127.

[19] M.P. Bendsøe, O. Sigmund, Material interpolation schemes in topology optimization, Archive of applied mechanics, 69 (1999) 635-654.

[20] Y. Xie, G.P. Steven, A simple evolutionary procedure for structural optimization, Computers \& structures, 49 (1993) 885-896.

[21] O. Querin, G. Steven, Y. Xie, Evolutionary structural optimisation (ESO) using a bidirectional algorithm, Engineering Computations, 15 (1998) 1031-1048.

[22] M.Y. Wang, X. Wang, D. Guo, A level set method for structural topology optimization, Computer methods in applied mechanics and engineering, 192 (2003) 227-246.

[23] G. Allaire, F. Jouve, A.-M. Toader, Structural optimization using sensitivity analysis and a level-set method, Journal of computational physics, 194 (2004) 363-393.

[24] V. Challis, A. Roberts, A. Wilkins, Design of three dimensional isotropic microstructures for maximized stiffness and conductivity, International Journal of Solids and Structures, 45 (2008) 41304146.

[25] Y. Wang, Z. Luo, N. Zhang, Q. Qin, Topological shape optimization of multifunctional tissue engineering scaffolds with level set method, Structural and Multidisciplinary Optimization, (2016) 1-15.

[26] X. Huang, A. Radman, Y. Xie, Topological design of microstructures of cellular materials for maximum bulk or shear modulus, Computational Materials Science, 50 (2011) 1861-1870.

[27] L. Xia, P. Breitkopf, Design of materials using topology optimization and energy-based homogenization approach in Matlab, Structural and Multidisciplinary Optimization, 52 (2015) 1229-1241. [28] O. Sigmund, Materials with prescribed constitutive parameters: an inverse homogenization problem, International Journal of Solids and Structures, 31 (1994) 2313-2329.

[29] W. Zhang, G. Dai, F. Wang, S. Sun, H. Bassir, Using strain energy-based prediction of effective elastic properties in topology optimization of material microstructures, Acta Mechanica Sinica, 23 (2007) 77-89.

[30] B. Niu, J. Yan, G. Cheng, Optimum structure with homogeneous optimum cellular material for maximum fundamental frequency, Structural and Multidisciplinary Optimization, 39 (2009) 115-132.

[31] P. Coelho, P. Fernandes, J. Guedes, H. Rodrigues, A hierarchical model for concurrent material and topology optimisation of three-dimensional structures, Structural and Multidisciplinary Optimization, 35 (2008) 107-115.

[32] P. Nakshatrala, D. Tortorelli, K. Nakshatrala, Nonlinear structural design using multiscale topology optimization. Part I: Static formulation, Computer Methods in Applied Mechanics and Engineering, 261 (2013) 167-176.

[33] S.A. Khanoki, D. Pasini, Multiscale design and multiobjective optimization of orthopedic hip implants with functionally graded cellular material, Journal of biomechanical engineering, 134 (2012) 031004.

[34] T.J.R. Hughes, J.A. Cottrell, Y. Bazilevs, Isogeometric analysis: CAD, finite elements, NURBS, exact geometry and mesh refinement, Computer methods in applied mechanics and engineering, 194 (2005) 4135-4195.

[35] J.A. Cottrell, T.J. Hughes, Y. Bazilevs, Isogeometric analysis: toward integration of CAD and FEA, John Wiley \& Sons, 2009.

[36] J. Yan, A. Korobenko, X. Deng, Y. Bazilevs, Computational free-surface fluid-structure interaction with application to floating offshore wind turbines, Computers \& Fluids, (2016).

[37] Y. Bazilevs, X. Deng, A. Korobenko, F.L. di Scalea, M. Todd, S. Taylor, Isogeometric Fatigue Damage Prediction in Large-Scale Composite Structures Driven by Dynamic Sensor Data, Journal of Applied Mechanics, 82 (2015) 091008. 
[38] X. Deng, A. Korobenko, J. Yan, Y. Bazilevs, Isogeometric analysis of continuum damage in rotation-free composite shells, Computer Methods in Applied Mechanics and Engineering, 284 (2015) 349-372.

[39] Y. Wang, D.J. Benson, A.P. Nagy, A multi-patch nonsingular isogeometric boundary element method using trimmed elements, Computational Mechanics, 56 (2015) 173-191.

[40] Y. Wang, D.J. Benson, Multi-patch nonsingular isogeometric boundary element analysis in 3D, Computer Methods in Applied Mechanics and Engineering, 293 (2015) 71-91.

[41] M.-C. Hsu, D. Kamensky, Y. Bazilevs, M.S. Sacks, T.J. Hughes, Fluid-structure interaction analysis of bioprosthetic heart valves: significance of arterial wall deformation, Computational mechanics, 54 (2014) 1055-1071.

[42] D. Schillinger, L. Dede, M.A. Scott, J.A. Evans, M.J. Borden, E. Rank, T.J. Hughes, An isogeometric design-through-analysis methodology based on adaptive hierarchical refinement of NURBS, immersed boundary methods, and T-spline CAD surfaces, Computer Methods in Applied Mechanics and Engineering, 249 (2012) 116-150.

[43] A. Diaz, O. Sigmund, Checkerboard patterns in layout optimization, Structural optimization, 10 (1995) 40-45.

[44] O. Sigmund, K. Maute, Topology optimization approaches, Structural and Multidisciplinary Optimization, 48 (2013) 1031-1055.

[45] K. Matsui, K. Terada, Continuous approximation of material distribution for topology optimization, International Journal for Numerical Methods in Engineering, 59 (2004) 1925-1944.

[46] Y.-D. Seo, H.-J. Kim, S.-K. Youn, Isogeometric topology optimization using trimmed spline surfaces, Computer Methods in Applied Mechanics and Engineering, 199 (2010) 3270-3296.

[47] B. Hassani, M. Khanzadi, S.M. Tavakkoli, An isogeometrical approach to structural topology optimization by optimality criteria, Structural and multidisciplinary optimization, 45 (2012) 223-233.

[48] L. Dedè, M.J. Borden, T.J. Hughes, Isogeometric analysis for topology optimization with a phase field model, Archives of Computational Methods in Engineering, 19 (2012) 427-465.

[49] Y. Wang, D.J. Benson, Isogeometric analysis for parameterized LSM-based structural topology optimization, Computational Mechanics, 57 (2016) 19-35.

[50] B. Hassani, E. Hinton, Homogenization and structural topology optimization: theory, practice and software, Springer Science \& Business Media, 2012.

[51] S.J. Hollister, N. Kikuchi, A comparison of homogenization and standard mechanics analyses for periodic porous composites, Computational Mechanics, 10 (1992) 73-95.

[52] P. Les, T. Wayne, The NURBS book, Springer Verlag, Berlin, (1997).

[53] C. De Boor, On calculating with B-splines, Journal of Approximation Theory, 6 (1972) 50-62.

[54] T.J.R. Hughes, The Finite Element Method: Linear Static and Dynamic Finite Element Analysis, Courier Corporation, 2000.

[55] E. Andreassen, A. Clausen, M. Schevenels, B.S. Lazarov, O. Sigmund, Efficient topology optimization in MATLAB using 88 lines of code, Structural and Multidisciplinary Optimization, 43 (2011) $1-16$.

[56] V. Rion, M. Bruyneel, Topology optimization of membranes made of orthotropic material, in: Collection of Papers from Prof. Nguyen Dang Hung's Former Students, Vietnam National UniversityHCM Publishing House, Vietnam, 2006.

[57] T.J.R. Hughes, J.A. Cottrell, Y. Bazilevs, Isogeometric analysis: CAD, finite elements, NURBS, exact geometry and mesh refinement, Computer methods in applied mechanics and engineering, 194 (2005) 4135-4195.

[58] Z. Luo, M.Y. Wang, S. Wang, P. Wei, A level set-based parameterization method for structural shape and topology optimization, International Journal for Numerical Methods in Engineering, 76 (2008) $1-26$.

[59] Z. Luo, L. Tong, Z. Kang, A level set method for structural shape and topology optimization using radial basis functions, Computers \& Structures, 87 (2009) 425-434. 
[60] S. Wang, M.Y. Wang, Radial basis functions and level set method for structural topology optimization, International journal for numerical methods in engineering, 65 (2006) 2060-2090.

[61] M.P. Bendsøe, O. Sigmund, Topology optimization: theory, methods and applications. , in, Springer, Berlin, 2003.

[62] T. Liu, S. Wang, B. Li, L. Gao, A level-set-based topology and shape optimization method for continuum structure under geometric constraints, Structural and Multidisciplinary Optimization, 50 (2014) 253-273.

[63] Y.-D. Seo, H.-J. Kim, S.-K. Youn, Shape optimization and its extension to topological design based on isogeometric analysis, International Journal of Solids and Structures, 47 (2010) 1618-1640.

[64] M. Zhou, B.S. Lazarov, F. Wang, O. Sigmund, Minimum length scale in topology optimization by geometric constraints, Computer Methods in Applied Mechanics and Engineering, 293 (2015) 266-282.

[65] O. Sigmund, Morphology-based black and white filters for topology optimization, Structural and Multidisciplinary Optimization, 33 (2007) 401-424.

[66] T.L. Gerstle, A.M. Ibrahim, P.S. Kim, B.T. Lee, S.J. Lin, A plastic surgery application in evolution: three-dimensional printing, Plastic and reconstructive surgery, 133 (2014) 446-451.

[67] A.C. Cameron, F.A. Windmeijer, An R-squared measure of goodness of fit for some common nonlinear regression models, Journal of Econometrics, 77 (1997) 329-342. 